Pacific

Journal of

Mathematics

\title{
ON A SPECIAL CLASS OF FIBRATIONS AND KÄHLER RIGIDITY
}

NiCKOLAS J. MiCHELACAKIS

Volume $219 \quad$ No. 2

April 2005 


\title{
ON A SPECIAL CLASS OF FIBRATIONS AND KÄHLER RIGIDITY
}

\author{
NiCKOLAS J. MiCHELACAKIS
}

\begin{abstract}
Let $A \mathscr{B}^{n}$ be the class of torsion-free, discrete groups that contain a normal, at most $\boldsymbol{n}$-step, nilpotent subgroup of finite index. We give sufficient conditions for the fundamental group of a fibration $F \rightarrow T \rightarrow B$, with base $B$ an infra-nilmanifold, to belong to $A_{\mathscr{B}}{ }^{n}$. Manifolds of this kind may, for example, appear as thin ends of nonpositively curved manifolds. We prove that if, in addition, we require that $T$ be Kähler, then $T$ possesses a flat Riemannian metric and the fundamental group $\pi_{1}(T)$ is necessarily a Bieberbach group. Further, we prove that a torsion-free, virtually polycyclic group that can be realised as the fundamental group of a compact, $K a ̈ h l e r ~ K(\pi, 1)$-manifold is necessarily Bieberbach.
\end{abstract}

\section{Introduction}

Torsion-free, discrete, cocompact subgroups of the group of affine motions of $\mathbb{R}^{n}$ were first studied by Bieberbach in 1912, and more recently by Charlap; they are called Bieberbach groups. They correspond precisely to the fundamental groups of compact manifolds endowed with a flat Riemannian metric [Charlap 1965], and such manifolds are finitely covered by flat tori [Bieberbach 1911].

L. Auslander [1960] and Lee and Raymond [1985] turned their attention to almost-Bieberbach groups, that is, torsion-free, discrete, cocompact subgroups of $G \rtimes C$, with $C$ a maximal, compact subgroup of Aut $G$ for $G$ a simply connected, nilpotent Lie group. They succeeded in generalising much of Bieberbach's work. Malcev's equivalence [1949] shows that torsion-free, finitely generated, nilpotent groups correspond precisely to the fundamental groups of nilmanifolds, that is, compact manifolds of the form $M=G / N$, where $G$ is a simply connected, nilpotent Lie group, and $N$ a discrete subgroup. Theorem 3.2 shows that almost-Bieberbach groups correspond to infra-nilmanifolds, compact manifolds of the form $G / \Gamma$ with $G$ as above and $\Gamma$ a discrete subgroup of $G \rtimes C$, where

MSC2000: primary 22E40; secondary 32Q15, 14R20.

Keywords: affinely flat manifold, (almost)-crystallographic, (almost)-Bieberbach group,

(almost)-torsion-free, (virtually) polycyclic group, nilpotent Lie group, discrete cocompact subgroups, lattice, Malcev completion, cohomology of groups, complex (Kähler) structure, group action, group representation, flat Riemannian manifold, (infra)-nilmanifold. 
$C$ is a maximal compact subgroup of Aut $G$. We denote by $\mathscr{A}_{\mathscr{B}^{n}}$ the class of almost-Bieberbach groups whose maximal, normal, nilpotent subgroup is at most $n$-step nilpotent. We shall say that a group $\Gamma$ admits an $n$-step almost-Bieberbach structure if and only if $\Gamma \in \mathscr{A}_{\mathscr{B}}{ }^{n}$ and its maximal normal nilpotent subgroup is $n$-step nilpotent.

(We know from [Gromov 1981] and [Wolf 1968] that, among finitely generated groups, virtually nilpotent groups are precisely those groups that have polynomial growth. For details and precise definitions, see those works or [Tits 1981].)

We employ algebraic methods to study closed manifolds that fibre over infranilmanifolds. If $F \rightarrow T \rightarrow B$ is such a fibration, where $F, T$ and $B$ are all acyclic, the long homotopy exact sequence reduces to a group extension of the form

$$
1 \longrightarrow \pi_{1}(F) \longrightarrow \pi_{1}(T) \longrightarrow \pi_{1}(B) \longrightarrow 1 .
$$

Manifolds of this type appear as thin ends of geometrically finite hyperbolic manifolds, which are an interesting subclass of nonpositively curved manifolds. More specifically, Apanasov and Xie [1997] proved that if $\Gamma \subset \mathscr{H}_{n} \rtimes U(n-1)$ is a torsionfree discrete group acting on the Heisenberg group $\mathscr{H}_{n}:=\mathbb{C}^{n-1} \times \mathbb{R}$, the orbit space $\mathscr{H}_{n} / \Gamma$ is a Heisenberg manifold of zero Euler characteristic and a vector bundle over a compact manifold. Further, this compact manifold is finitely covered by a nilmanifold which is either a torus or a torus bundle over a torus. This generalises earlier results on almost flat manifolds concerning lattices in $\mathscr{H}_{n} \rtimes U(n-1)$ [Gromov 1978; Buser and Karcher 1981].

As mentioned above, groups in $\mathscr{A} \mathscr{B}^{n}$ correspond to infra-nilmanifolds. In Section 2 we study extensions of the form

$$
1 \longrightarrow G \longrightarrow \Gamma \longrightarrow K \longrightarrow 1
$$

with $K \in \mathscr{A}^{n}$, to provide sufficient conditions under which $\Gamma$ belongs to $\mathscr{A} \mathscr{B}^{n}$. In particular, Proposition 2.2 guarantees the existence of an almost-Bieberbach structure on $\Gamma$ provided $G$ is a normal subgroup of $\Gamma$ in a precise way. Proposition 2.4 does the same provided $G$ lies in $\mathscr{A} \mathscr{B}^{n}$ and the action of $K$ on $G$ respects some suitable minimal conditions.

In Section 3 we use the Johnson-Rees characterisation of fundamental groups of flat, Kähler [Johnson and Rees 1991], and projective [Johnson 1990] manifolds, and apply the Benson-Gordon theorem [1988] for the existence of a Kähler structure on a compact nilmanifold to show, in Theorem 3.3, that the existence of a Kähler structure on a special fibration as above implies the existence of a flat Riemann metric on $T$. In particular, in $\mathscr{A}_{\mathscr{B}^{n}}$, the classes of fundamental groups of Kähler and projective manifolds coincide, as shown in Corollary 3.4. Further, as a consequence of the Lefschetz hyperplane theorem and Bertini's theorem, this 
is a subclass of the class of fundamental groups of compact, closed, nonsingular projective surfaces.

Finally, in Section 4, we use a structure theorem concerning virtually polycyclic groups, proved in [Dekimpe and Igodt 1994], together with the results in [Arapura and Nori 1999], to prove, in Theorem 4.2, that a torsion-free, virtually polycyclic group can be realised as the fundamental group of a $\mathrm{K}(\pi, 1)$-compact, Kähler manifold if and only if it is Bieberbach of a special kind, namely, its operator homomorphism is essentially complex.

\section{Group extensions}

A group $N$ is said to be nilpotent if its upper central series

$$
1=N_{0} \triangleleft N_{1}=Z(N) \triangleleft N_{2} \triangleleft \cdots,
$$

defined by $N_{i+1} / N_{i}=Z\left(N / N_{i}\right)$, is finite. If $n$ is the smallest integer such that $N_{n}=N$, then $N$ is said to be $n$-step nilpotent. We shall say that a finitely generated, torsion-free group $\Gamma$ admits an ( $n$-step) almost-Bieberbach group structure if it can be written as an extension of a finitely generated, ( $n$-step) nilpotent group $N$ by a finite group $\Phi$. Notice that, given such a torsion-free, finitely generated, nilpotent group, its quotients $N_{i+1} / N_{i}$ are of a special form, namely $N_{i+1} / N_{i} \cong \mathbb{Z}^{i_{j}}$.

Lemma 2.1. Let $\Gamma$ fit in an extension

$$
0 \longrightarrow \mathbb{Z}^{m} \longrightarrow \Gamma \stackrel{p}{\longrightarrow} G \longrightarrow 1
$$

where the torsion-free group $G$ has an n-step nilpotent, normal subgroup $N$ of finite index and $\mathbb{Z}^{m}$ a trivial $N$-module. Then $\Gamma \in \mathscr{A}_{\mathbb{B}^{n+1}}$.

Proof. Let $G$ be defined by the extension

$$
1 \longrightarrow N \longrightarrow G \longrightarrow \Phi \longrightarrow 1,
$$

with $N n$-step nilpotent, $\Phi$ finite and $\phi: \Phi \rightarrow$ Out $N$ the operator homomorphism. Consider $\bar{\Gamma}:=p^{-1}(N)$. Then the extension

$$
0 \longrightarrow \mathbb{Z}^{m} \longrightarrow \bar{\Gamma} \stackrel{p}{\longrightarrow} N \longrightarrow 1
$$

is central, which implies that $\bar{\Gamma}$ is at most $(n+1)$-step nilpotent. The proof is completed by the observation that $\bar{\Gamma}=p^{-1}(N) \triangleleft \Gamma$ and $\Gamma / \bar{\Gamma} \cong\left(\Gamma / \mathbb{Z}^{m}\right) /\left(\bar{\Gamma} / \mathbb{Z}^{m}\right) \cong$ $G / N \cong \Phi$. Notice that $\Gamma$ is torsion-free since so are $\mathbb{Z}^{m}$ and $G$.

We now turn our attention to the fibre of the fibration $F \rightarrow T \rightarrow B$ to prove the following: 
Proposition 2.2. Let $\Gamma$ be a torsion-free extension

$$
1 \longrightarrow K \longrightarrow \Gamma \stackrel{p}{\longrightarrow} G \longrightarrow 1
$$

of a finitely generated group $K$ by a group $G$ admitting an $n$-step nilpotent, almostBieberbach structure such that $\operatorname{Im} c$ is finite, where $c: \Gamma \rightarrow$ Aut $K$ denotes the conjugation map. Then $\Gamma \in \mathscr{A}_{\mathscr{B}^{n+1}}$.

Proof. Since $G$ admits an $n$-step almost-Bieberbach structure there is a short exact sequence

$$
1 \longrightarrow N \longrightarrow G \longrightarrow \Phi \longrightarrow 1
$$

where $N$ is $n$-step nilpotent, $\Phi$ is finite, and $\phi: \Phi \rightarrow$ Out $N$ is the operator homomorphism. Let $\hat{\Gamma}:=p^{-1}(N)$. Then $\hat{\Gamma}$ fits in a short exact sequence

$$
1 \longrightarrow K \longrightarrow \hat{\Gamma} \stackrel{p}{\longrightarrow} N \longrightarrow 1,
$$

where we denote by $\bar{c}$ the restriction of the conjugation map $c: \Gamma \rightarrow$ Aut $K$ to $\hat{\Gamma}$. Let $\bar{\Gamma}:=\operatorname{Ker} \bar{c}$, which is nonempty since $\Gamma$ is infinite. Then the extension

$$
1 \longrightarrow \bar{\Gamma} \cap K \longrightarrow \bar{\Gamma} \longrightarrow p(\bar{\Gamma}) \longrightarrow 1
$$

is central, with $p(\bar{\Gamma}) \triangleleft N$, and therefore itself nilpotent. This means that $\bar{\Gamma} \cap K$ is a finitely generated, torsion-free, abelian group and $\bar{\Gamma}$ is at most $(n+1)$-step nilpotent. The proof is completed by observing that the normal subgroup $\bar{\Gamma}$ of $\hat{\Gamma}$ has finite index in $\hat{\Gamma}$, since $\operatorname{Im} \bar{c}$ is finite.

The group Aut $K$, for $K$ a Bieberbach group, is not necessarily finite. For an example, see [Charlap 1986, p. 219]. It does, then, make sense to check what happens if the fibre admits an $n$-step almost-Bieberbach structure. But first:

Proposition 2.3. Let $\Gamma$ be a torsion-free extension

$$
1 \longrightarrow K \longrightarrow \Gamma \stackrel{p}{\longrightarrow} \mathbb{Z}^{n} \longrightarrow 0
$$

of a Bieberbach group $K$ by a free abelian group of rank $n$, such that $\mathbb{Z}^{m} \subseteq Z(\Gamma)$, where $\mathbb{Z}^{m}$ is the translation subgroup of $K$ and $Z(\Gamma)$ the center of $\Gamma$. Then $\Gamma \in$ $\mathscr{A B}^{2}$.

Proof. First observe that $\mathbb{Z}^{m} \triangleleft \Gamma$, since $\mathbb{Z}^{m} \leq Z(\Gamma)$. We therefore have a short exact sequence

$$
1 \longrightarrow K / \mathbb{Z}^{m} \longrightarrow \Gamma / \mathbb{Z}^{m} \stackrel{p}{\longrightarrow} \mathbb{Z}^{n} \longrightarrow 0
$$

where $K / \mathbb{Z}^{m}$ is isomorphic to $F$, the finite holonomy group of $K$. We distinguish two cases: 
(i) Assume that (2-3) is a central extension. Then choose $Q:=\left(\mathbb{Z}^{n-1} \times k \mathbb{Z}\right) \triangleleft \mathbb{Z}^{n}$ of index $k=|F|$, with $|F|$ the exponent of $F$. Let $\Gamma^{\prime}:=p^{-1}(Q)$. Then $\Gamma^{\prime}$ fits in a short exact sequence

$$
1 \longrightarrow F \longrightarrow \Gamma^{\prime} \stackrel{p}{\longrightarrow} Q \longrightarrow 0
$$

that splits as a direct product. By construction, $\Gamma^{\prime}=(F \times Q) \triangleleft \Gamma / \mathbb{Z}^{m}$ is of finite index. Let $q: \Gamma \rightarrow \Gamma / \mathbb{Z}^{m}$ be the identification map. The free abelian group $Q$ imbeds as a normal subgroup of $\Gamma^{\prime} \triangleleft \Gamma / \mathbb{Z}^{m}$ and so also, because $\operatorname{Aut}(F \times Q)=$ Aut $F \times$ Aut $Q$, as a normal subgroup of $\Gamma / \mathbb{Z}^{m}$. Let $\tilde{\Gamma}:=q^{-1}(Q)$ and $\hat{\Gamma}:=q^{-1}\left(\Gamma^{\prime}\right)$; then $Q \cong \tilde{\Gamma} / \mathbb{Z}^{m}$ and $F \times Q \cong \hat{\Gamma} / \mathbb{Z}^{m}$. Since $Q \leq \mathbb{Z}^{n}$, it acts on $\mathbb{Z}^{m}$ in the same way as $\mathbb{Z}^{n}$, namely trivially. So $\tilde{\Gamma}$ is 2-step nilpotent normal in $\Gamma$. One can further check that its index $|\Gamma / \tilde{\Gamma}|$ in $\Gamma$ is finite, because $|\Gamma / \tilde{\Gamma}|=|\Gamma / \hat{\Gamma}| \cdot|\hat{\Gamma} / \tilde{\Gamma}|=\left|\mathbb{Z}^{n} / Q\right| \cdot|F|$. This completes the proof in this case.

(ii) Assume that the sequence (2-3) is not central, and let $c: \Gamma / \mathbb{Z}^{m} \rightarrow$ Aut $F$ be the conjugation map. Since $F$ is finite and $\Gamma / \mathbb{Z}^{m}$ infinite, the kernel of $c$ is nontrivial. Let $\bar{\Gamma}:=\operatorname{Ker} c \triangleleft \Gamma / \mathbb{Z}^{m}$, let $\bar{F}:=F \cap \bar{\Gamma}$, and let $\bar{Q}:=p(\bar{\Gamma})$. Then the extension

$$
1 \longrightarrow \bar{F} \longrightarrow \bar{\Gamma} \longrightarrow \bar{Q} \longrightarrow 0
$$

with $\bar{Q} \triangleleft \mathbb{Z}^{n}$ (so that $\bar{Q} \cong \mathbb{Z}^{\rho}$ for some $\rho \leq n$ ) belongs to the previous case. The result now follows, since $\bar{\Gamma}$ has finite index in $\Gamma$.

Proposition 2.4. Let $\Gamma$ be a torsion-free extension

$$
1 \longrightarrow K \longrightarrow \Gamma \stackrel{p}{\longrightarrow} G \longrightarrow 1,
$$

where $K$ and $G$ admit $m$-step and n-step almost-Bieberbach structures, respectively. If $Z\left(L / L_{i}\right) \subseteq Z\left(\Gamma / L_{i}\right)$, where $\left\{L_{i}\right\}_{i}$ is the upper central series of an $m$-step nilpotent, normal subgroup $L$ of finite index in $K$, then $\Gamma \in \mathscr{A}_{\mathbb{B}^{n+m}}$.

Proof. We first check inductively that $L_{i} \triangleleft \Gamma$. This is clear for $i=1$. Assume it is true for $i$ and let $q_{i}: L \rightarrow L / L_{i}$ be the identification map, where

$$
L_{i+1}=q^{-1}\left(Z\left(L / L_{i}\right)\right),
$$

so that $L_{i+1} / L_{i} \cong Z\left(L / L_{i}\right)$. Then $L_{i+1} / L_{i} \triangleleft Z\left(\Gamma / L_{i}\right)$ and $L_{i+1} \triangleleft \Gamma$. The rest of the proof also follows by induction, first on $m$ and then on $n$. The group $G$ is of the form

$$
1 \longrightarrow N \longrightarrow G \longrightarrow \Phi \longrightarrow 1,
$$

where $N$ is $n$-step nilpotent and $\Phi$ finite. By letting $\hat{\Gamma}:=p^{-1}(N)$ we get a sequence

$$
1 \longrightarrow K \longrightarrow \hat{\Gamma} \longrightarrow N \longrightarrow 1 \text {. }
$$


The case $m=n=1$ follows from Proposition 2.3. Assuming the theorem is true for some $m$ and $n=1$, we shall show it is true for $m+1$ and $n=1$. If $K$ is of the form

$$
1 \longrightarrow L \longrightarrow K \longrightarrow F \longrightarrow 1,
$$

where $L$ is $m$-step nilpotent and $F$ finite, consider $L_{1}=Z(L)$. The conditions of the theorem ensure that $L_{1}=Z(L) \cong \mathbb{Z}^{\rho} \triangleleft \Gamma$ for some positive integer $\rho$. This gives a short exact sequence

$$
1 \longrightarrow K / \mathbb{Z}^{\rho} \longrightarrow \hat{\Gamma} / \mathbb{Z}^{\rho} \longrightarrow \mathbb{Z}^{v} \longrightarrow 0,
$$

with $v>0$. Then $\left\{L_{i} / L_{1}\right\}_{i}$ is the upper central series of $L / L_{1}$ and $\hat{\Gamma} / \mathbb{Z}^{\rho}$ admits an $(n+1)$-step almost-Bieberbach structure by the induction hypothesis. $\hat{\Gamma}$ fits into a central short exact sequence

$$
0 \longrightarrow \mathbb{Z}^{\rho} \longrightarrow \hat{\Gamma} \longrightarrow \hat{\Gamma} / \mathbb{Z}^{\rho} \longrightarrow 1 .
$$

Lemma 2.1 now applies to prove that $\hat{\Gamma}$, and therefore $\Gamma$, admit an $(n+2)$-step almost-Bieberbach structure. Now assume the theorem is true for all $m$ and $n$ up to a certain value. We complete the proof by showing it holds for all $m$ and $n+1$. If $\left\{N_{i}\right\}_{i}$ is the upper central series of some $(n+1)$-step nilpotent $N$, define $\bar{\Gamma}:=p^{-1}\left(N_{n}\right)$. Then $\bar{\Gamma}$ fits in

$$
1 \longrightarrow K \longrightarrow \bar{\Gamma} \longrightarrow N_{n} \longrightarrow 1
$$

and admits an $(n+m)$-step almost-Bieberbach structure. Also there is a positive integer $\mu$ such that the sequence

$$
1 \longrightarrow \bar{\Gamma} \longrightarrow \hat{\Gamma} \longrightarrow \bar{\Gamma} / \hat{\Gamma} \cong \mathbb{Z}^{\mu} \longrightarrow 0
$$

is exact. The induction argument on the fibre implies that $\hat{\Gamma} \in \mathscr{A} \mathscr{B}^{n+m+1}$, and so $\Gamma \in \mathscr{A}_{\mathscr{B}^{n+m+1}}$ too.

\section{Almost-Bieberbach groups and Kähler structures}

Let Aut $G$ denote the group of automorphisms of a simply connected Lie group $G$. We shall be concerned with discrete subgroups $\Gamma$ of Aut $G$ that act properly discontinuously on $G$.

A group $\Gamma$ is said to be crystallographic if it is a cocompact, discrete subgroup of $\mathbb{R}^{n} \rtimes O(n) \subset \operatorname{Aff}\left(\mathbb{R}^{n}\right)$, where $O(n)$ is the maximal compact subgroup of $\operatorname{GL}(n, \mathbb{R})$ and $\operatorname{Aff}\left(\mathbb{R}^{n}\right)$ is the group of Euclidean motions of $\mathbb{R}^{n}$. It is a Bieberbach crystallographic group if it is torsion-free as well. Bieberbach groups are precisely the fundamental groups of compact, complete Riemannian manifolds that are flat (locally isometric to Euclidean space), as first proved in [Bieberbach 1911]. An alternative characterisation of flat Riemannian manifolds is that in such manifolds, 
transition maps can be extended to elements of $\mathbb{R}^{n} \rtimes O(n)$. Charlap [1965] classified these manifolds, up to connection-preserving diffeomorphisms, by associating to a manifold $M$ a short exact sequence

$$
1 \longrightarrow \Lambda \longrightarrow G \longrightarrow \Phi \longrightarrow 1
$$

in which the holonomy group $\Phi$ of $M$ is finite and $\Lambda \cong \mathbb{Z}^{n}$ is the translation subgroup of $\Gamma \cong \pi_{1}(M)$, a torsion-free, discrete, cocompact subgroup of $\mathbb{R}^{n} \rtimes O(n) \subset$ $\operatorname{Aff}\left(\mathbb{R}^{n}\right)$.

More generally, if $G$ is a simply connected, nilpotent Lie group, we consider a maximal compact subgroup $C \subseteq$ Aut $G$. A cocompact, discrete subgroup $\Gamma$ of $G \rtimes C$ is called an almost-crystallographic group, and if torsion-free it is called almost-Bieberbach. The quotient $G / \Gamma$ is called an infra-nilmanifold, and if $\Gamma \subseteq G$ it is a nilmanifold.

Most of Bieberbach's work has been generalised to the nilpotent case in [Auslander 1960] and [Lee and Raymond 1985]:

Theorem 3.1 (Auslander). Let $\Gamma \subseteq G \rtimes$ Aut $G$ be an almost-crystallographic group, where $G$ is a connected, simply connected, nilpotent Lie group. Then $(\Gamma \cap G) \triangleleft \Gamma$ is a cocompact lattice in $G$, and $\Gamma /(\Gamma \cap G)$ is finite.

Parts of the statement of the following theorem can already be found in [Lee and Raymond 1985]. We simplify the proof.

Theorem 3.2. $\Gamma$ is almost-crystallographic if and only if it is of the form

$$
1 \longrightarrow N \longrightarrow \Gamma \longrightarrow \Phi \longrightarrow 1,
$$

with $N$ finitely generated, torsion-free, maximal nilpotent, and $\Phi$ finite.

Proof. If $\Gamma \subseteq G \rtimes$ Aut $G$ is an almost-Bieberbach group, Theorem 3.1 says that $N=\Gamma \cap G$ is a maximal nilpotent, normal subgroup of $\Gamma$ of finite index, and finitely generated because it is a discrete subgroup of the nilpotent group $G$. To prove the converse, given an extension like the one in the statement of the theorem, with abstract kernel $\phi: \Phi \rightarrow$ Out $N$, consider the extension of the Malcev completion $\mathcal{N}$ of $N$,

$$
1 \longrightarrow \mathcal{N} \longrightarrow S(\Gamma) \longrightarrow \Phi \longrightarrow 1,
$$

with abstract kernel $\psi: \Phi \stackrel{\phi}{\rightarrow}$ Out $N \rightarrow$ Out $\mathcal{N}$. The claim is that there is exactly one extension of $\mathcal{N}$ by $\Phi$, namely $\mathcal{N} \hookrightarrow \mathcal{N}_{\rtimes_{\hat{\psi}}} \Phi$, where $\hat{\psi}: \Phi \rightarrow$ Aut $\mathcal{N}$ is a lifting morphism of $\psi$.

Since $Z(\mathcal{N})$ is a vector space and $\Phi$ is finite, $H^{3}(\Phi, Z(\mathcal{N}))$ vanishes and by [Mac Lane 1963, Theorem 8.7] the abstract kernel $[\Phi, \mathcal{N}, \psi]$ has an extension. Furthermore, [Mac Lane 1963, Theorem 8.8] says that this extension is unique 
because the set $H^{2}(\Phi, Z(\mathcal{N}))$ parametrizing all congruence classes of such extensions is null, for the same reason. So we know that there is precisely one extension $\mathcal{N} \hookrightarrow \Gamma \rightarrow \Phi$. If we can further show that $\psi$ has a lifting morphism $\hat{\psi}: \Phi \rightarrow$ Aut $\mathcal{N}$, then $\Gamma \cong \mathcal{N}_{\hat{\psi}} \Phi$. To this end, we apply induction on the nilpotency class of $\mathcal{N}$. If $\mathcal{N}$ is 1 -step nilpotent then $\mathcal{N} \cong Z(\mathcal{N})$ and the result is obvious. If $\mathcal{N}$ is $c$-step nilpotent, consider the inverse image under the natural projection $q:$ Aut $\mathcal{N} \rightarrow$ Out $\mathcal{N}$ of the finite group $\psi(\Phi)$. This gives birth to a short exact sequence $\operatorname{Inn} \mathcal{N} \hookrightarrow$ $q^{-1}(\psi(\Phi)) \rightarrow \psi(\Phi)$ with Inn $\mathcal{N} \cong \mathcal{N} / Z(\mathcal{N})$ fulfilling the induction hypothesis. We can thus find a splitting morphism $s: \psi(\Psi) \rightarrow q^{-1}(\psi(\Phi))<$ Aut $\mathcal{N}$. But now, $s \circ \psi$ is the lifting we were looking for, completing the proof. We thus have the commutative diagram

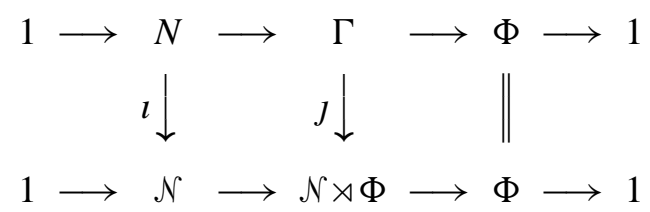

The map $J$, with $J(n, g)=(l(n), g)$, embeds $\Gamma$ as a discrete, cocompact subgroup of the disconnected Lie group $S(\Gamma)$, proving the theorem.

Given a short exact sequence $\mathbb{Z}^{2 n} \hookrightarrow \Gamma \rightarrow \Phi$ with operator homomorphism $\phi: \Phi \rightarrow$ Aut $\mathbb{Z}^{2 n}$, we say $\phi$ is essentially complex if there is a complex structure for the $\Phi$-module $\mathbb{Z}^{2 n} \otimes \mathbb{R}$, that is, a map $t \in \operatorname{End}_{\mathbb{R}[\Phi]}\left(\mathbb{Z}^{2 n} \otimes \mathbb{R}\right)$ such that $t^{2}=-1$. In other words, $\phi: \Phi \rightarrow$ Aut $\mathbb{Z}^{2 n}$ is essentially complex if $\operatorname{Im} \phi \subseteq \mathrm{GL}_{\mathbb{C}}\left(\left(\mathbb{Z}^{2 n} \otimes \mathbb{R}\right)^{t}\right)$, with

$$
\mathrm{GL}_{\mathbb{C}}\left(\left(\mathbb{Z}^{2 n} \otimes \mathbb{R}\right)^{t}\right):=\left\{m \in \mathrm{GL}_{\mathbb{R}}\left(\mathbb{Z}^{2 n} \otimes \mathbb{R}\right) \text { such that } m t=t m\right\} .
$$

Theorem 3.3. Let $\Gamma$ be the torsion-free extension

$$
1 \longrightarrow N \longrightarrow \Gamma \longrightarrow \Phi \longrightarrow 1,
$$

where $N$ is a torsion-free, finitely generated maximal nilpotent group and $\Phi$ is a finite group. Then there is a compact Kähler $K(\Gamma, 1)$-manifold $M$ if and only if $N \cong \mathbb{Z}^{2 n}$ and the operator homomorphism $\phi: \Phi \rightarrow$ Aut $N$ is essentially complex.

Proof. By Theorem 3.2 there is a connected, simply connected Lie group $G$ such that $\Gamma$ is a torsion-free, discrete, cocompact subgroup of $G \rtimes$ Aut $G$. Since $M$ is a $\mathrm{K}(\Gamma, 1)$-manifold, its universal covering is homeomorphically equivalent to $G$ and $M \cong G / \Gamma$. The hypotheses on $N$ say that $G$ contains $N \hookrightarrow \Gamma$ as a discrete cocompact subgroup. Then $\hat{M} \cong G / N$ is a compact $\mathrm{K}(N, 1)$-nilmanifold that covers $M$ in a finite, unramified way. Because the Kähler condition is local, the fact that $M$ admits a Kähler structure implies that $\hat{M}$ also admits a Kähler structure. The Benson-Gordon theorem says that this can happen only if $N \cong \mathbb{Z}^{2 n}$, forcing 
the finite cover $\hat{M}$ of $M$ to be holomorphically equivalent to the complex torus $\mathbb{C}^{n} / \mathbb{Z}^{2 n}$. The converse is settled by [Johnson and Rees 1991, Theorem 3.1].

Let $\mathscr{B}_{\mathscr{K}}$ be the class of groups that can be realised as fundamental groups of compact, Kähler manifolds whose underlying Riemannian structure is flat, and $\mathscr{B}_{\mathscr{P}} \subseteq \mathscr{B}_{\mathscr{K}}$ the subclass consisting of groups that can be realised as fundamental groups of complex projective varieties. Let $\mathscr{A}_{\mathscr{B}} \mathscr{K}$ denote the class of groups that can be realised as fundamental groups of compact nilmanifolds; that is, compact manifolds of the form $G / \Gamma$, where $G$ is a simply connected, nilpotent Lie group and $\Gamma$ a discrete subgroup admitting a Kähler structure, and let $\mathscr{A}_{\mathscr{B} \mathscr{P}} \subseteq \mathscr{A}_{\mathscr{B} \mathscr{K}}$ be the subclass consisting of groups that can be realised as fundamental groups of complex projective nilvarieties.

Corollary 3.4. (1) $\mathscr{A} \mathscr{B}_{\mathscr{K}} \equiv \mathscr{B}_{\mathscr{K}} \equiv \mathscr{B}_{\mathscr{P}} \equiv \mathscr{A} \mathscr{\mathscr { P }}$.

(2) Every group in $\mathscr{A}_{\mathscr{B}} \mathscr{x}$ is the fundamental group of a smooth, compact, complex algebraic surface.

Proof. (1) The first equality follows directly from Theorem 3.3 and [Johnson and Rees 1991, Theorem 3.1]. The second is [Johnson 1990, Corollary 4.3], while the third stems from the first two together with the inclusion $A_{\mathscr{B}} \subseteq \mathscr{A}_{\mathscr{P}} \mathscr{K}$.

(2) If $M$ is a smooth projective manifold, then by Bertini's theorem there is a smooth hyperplane section $M_{(n-1)}$. By the Lefschetz hyperplane theorem [Milnor 1963], $\pi_{l}\left(M, M_{(n-1)}\right)=0$ for $l<n$, so $M$ and $M_{(n-1)}$ have isomorphic fundamental groups if $n \geq 3$.

We now combine Proposition 2.2, Proposition 2.4 and Theorem 3.3:

Theorem 3.5. If the Kähler manifold $T$ is the total space of a fibration $F \rightarrow T \rightarrow B$ over an infra-nilmanifold $B$ with aspherical fibre $F$ and if the short exact sequence

$$
1 \longrightarrow \pi_{1}(F) \longrightarrow \pi_{1}(T) \longrightarrow \pi_{1}(B) \longrightarrow 1
$$

of their respective fundamental groups satisfies the conditions of either Proposition 2.2 or Proposition 2.4, then $T$ admits a flat Riemannian metric.

\section{Virtually polycyclic groups and Kähler rigidity}

An affinely flat manifold is an $n$-manifold endowed with an atlas whose transition maps can be extended to elements of $\operatorname{Aff}\left(\mathbb{R}^{n}\right)=\mathbb{R}^{n} \rtimes \mathrm{GL}(n, \mathbb{R})$. A torsion-free group $\Gamma$ is virtually polycyclic if it has a subgroup $\Gamma_{0}$ of finite index which is polycyclic, that is, one that admits a finite composition series $\Gamma_{0} \supseteq \Gamma_{1} \supseteq \Gamma_{2} \supseteq$ $\cdots \supseteq \Gamma_{n}=1$ such that $\Gamma_{i} / \Gamma_{i+1} \cong \mathbb{Z}$ for all $i$. The number $n$ is an invariant, called the rank of $\Gamma$. Groups in $\mathscr{A}^{n}{ }^{n}$ are obviously virtually polycyclic. Auslander [1964] has conjectured that the fundamental group of a compact, complete, affinely 
flat manifold has to be virtually polycyclic. Milnor [1977] has shown that torsionfree, virtually polycyclic groups can be realised as fundamental groups of complete affinely flat manifolds. On the other hand, Johnson [1976] has proved that torsionfree, virtually polycyclic groups can be realised as fundamental groups of compact $\mathrm{K}(\pi, 1)$-manifolds. However, contrary to the Bieberbach case, Benoist [1992] has given an example of a 10-step nilpotent group of rank 11, proving that it is not always possible to do both!

If $\Gamma$ is a virtually polycyclic group, the Fitting group of $\Gamma$, denoted $\operatorname{Fitt}(\Gamma)$, is the unique maximal normal subgroup of $\Gamma$. The closure Fitt $(\Gamma)$ of the Fitting group of a group $\Gamma$ is the maximal normal subgroup of $\Gamma$ containing $\operatorname{Fitt}(\Gamma)$ as a normal subgroup of finite index. The basic property of $\overline{\operatorname{Fitt}(\Gamma)}$ is that it leaves the quotient $\Gamma / \overline{\operatorname{Fitt}(\Gamma)}$ with no finite, normal subgroup in it - in other words, almost-torsionfree. In [Dekimpe and Igodt 1994] it is proved that if $\Gamma$ is a finitely generated virtually nilpotent group then $\Gamma$ is almost-torsion-free if and only if $\overline{\operatorname{Fitt}(\Gamma)}$ is almost-crystallographic.

If $N$ is a torsion-free, finitely generated, $c$-step nilpotent group, then to any extension

$$
N \hookrightarrow \Gamma \stackrel{p}{\rightarrow} Q
$$

with abstract kernel $\psi: Q \rightarrow$ Out $N$ we can inductively associate $c$ morphisms $\psi_{i}: Q \rightarrow \operatorname{Aut}\left(N_{i+1} / N_{i}\right)$, where $N_{i+1} / N_{i}=Z\left(N / N_{i}\right)$. Now if $q \in \Gamma$ is such that $p(q)$ has finite order in $Q$, and $\langle q, N\rangle$ is nilpotent, then $p(q) \in \bigcap_{1}^{c} \operatorname{Ker} \psi_{i}$. Conversely, if $q \in \Gamma$ is such that $p(q) \in \bigcap_{1}^{c} \operatorname{Ker} \psi_{i}$, then $\langle q, N\rangle$ is nilpotent in $\Gamma$.

We shall use the following lemma, which is half of [Dekimpe and Igodt 1994, Theorem 2.2]. For completeness, we write a proof here.

Lemma 4.1. Let $\Gamma$ be a virtually polycyclic group. If $\Gamma$ is almost-torsion-free, $\operatorname{Fitt}(\Gamma)$ is torsion-free maximal nilpotent in $\Gamma$.

Proof. Since $\Gamma$ is polycyclic-by-finite, $\operatorname{Fitt}(\Gamma)$ is finitely generated nilpotent. Therefore its torsion set is a finite characteristic subgroup of $\operatorname{Fitt}(\Gamma)$, and thus normal in $\Gamma$, and hence trivial since $\Gamma$ is almost torsion-free. So, $\Gamma$ fits in an extension

$$
1 \longrightarrow \operatorname{Fitt}(\Gamma) \longrightarrow \Gamma \stackrel{p}{\longrightarrow} Q \longrightarrow 1
$$

with $\operatorname{Fitt}(\Gamma)$ torsion-free and $Q$ abelian-by-finite, say $A \hookrightarrow Q \stackrel{j}{\rightarrow} F$. Now let $q \in \Gamma$ be such that $N:=\langle q, \operatorname{Fitt}(\Gamma)\rangle$ is nilpotent, and look at $p(N)$. If $p(N) \cap A \neq\{1\}$ then $p^{-1}(p(N) \cap A)$ is normal in $\Gamma$ since $(p(N) \cap A) \triangleleft A$ is nilpotent as a subgroup of $N$. Thus, $p^{-1}(p(N) \cap A) \subseteq \operatorname{Fitt}(\Gamma)$ and $p(N) \cap A=\{1\}$, a contradiction. We deduce that $p(N) \cong j(p(N)) \subseteq F$, and hence that $p(q)$ is of finite order in $Q$. The discussion preceding the theorem shows that $p(q) \in \bigcap_{1}^{c}\left(\psi_{i}\right) \cap p(N)$, where $\psi_{i}$ are the morphisms associated with (4-1), which is a finite group since 
$F$ is finite; therefore $q \in \overline{\operatorname{Fitt}(\Gamma)}$. But since $\Gamma$ is almost torsion-free, $\overline{\operatorname{Fitt}(\Gamma)}$ is almost crystallographic and $\operatorname{Fitt}(\overline{\operatorname{Fitt}(\Gamma)})=\operatorname{Fitt}(\Gamma)$ is maximal nilpotent in $\overline{\operatorname{Fitt}(\Gamma)}$, implying $q \in \operatorname{Fitt}(\Gamma)$, a contradiction.

Theorem 4.2. Let $\Gamma$ be a torsion-free, virtually polycyclic group. Then $\Gamma$ can be realised as the fundamental group of a $K(\pi, 1)$ compact, Kähler manifold if and only if $\Gamma$ is Bieberbach with essentially complex operator homomorphism.

Proof. The converse is the second half of Theorem 3.3. For the direct statement, observe that since $\Gamma$ is torsion-free, it is almost-torsion-free. Thus, by Lemma 4.1, Fitt $(\Gamma)$ is torsion-free maximal nilpotent in $\Gamma$, and $\Gamma$ fits in a short exact sequence of the form

$$
1 \longrightarrow \operatorname{Fitt}(\Gamma) \longrightarrow \Gamma \stackrel{p}{\longrightarrow} Q \longrightarrow 1,
$$

where $Q$ is abelian-by-finite. Since $\Gamma$ is Kähler, by [Arapura and Nori 1999], there exists a nilpotent subgroup $\Delta \subseteq \Gamma$ of finite index. But $\Delta$ is necessarily contained in $\operatorname{Fitt}(\Gamma)$, so $Q$ is finite, and Theorem 3.3 completes the proof with $N=\operatorname{Fitt}(\Gamma)$.

Provided that the Auslander conjecture is true, Theorem 4.2 would immediately imply:

Conjecture 4.3. If a Kähler manifold $T$ is the total space of a fibration $F \rightarrow T \rightarrow B$ where both the base $B$ and the fibre $F$ are infra-nilmanifolds, then $T$ admits $a$ Riemann flat structure.

\section{References}

[Apanasov and Xie 1997] B. Apanasov and X. Xie, "Geometrically finite complex hyperbolic manifolds", Internat. J. Math. 8:6 (1997), 703-757. MR 99e:57051 Zbl 0912.53027

[Arapura and Nori 1999] D. Arapura and M. Nori, "Solvable fundamental groups of algebraic varieties and Kähler manifolds", Compositio Math. 116:2 (1999), 173-188. MR 2000k:14018 Zbl 0971.14020

[Auslander 1960] L. Auslander, "Bieberbach's theorems on space groups and discrete uniform subgroups of Lie groups", Ann. of Math. (2) 71 (1960), 579-590. MR 22 \#12161 Zbl 0099.25602

[Auslander 1964] L. Auslander, "The structure of complete locally affine manifolds", Topology 3:suppl. 1 (1964), 131-139. MR 28 \#4463 Zbl 0136.43102

[Auslander and Johnson 1976] L. Auslander and F. E. A. Johnson, "On a conjecture of C. T. C. Wall”, J. London Math. Soc. (2) 14:2 (1976), 331-332. MR 54 \#11341 Zbl 0364.22008

[Benoist 1992] Y. Benoist, "Une nilvariété non affine", C. R. Acad. Sci. Paris Sér. I Math. 315:9 (1992), 983-986. MR 93j:22008 Zbl 0776.57010

[Benson and Gordon 1988] C. Benson and C. S. Gordon, "Kähler and symplectic structures on nilmanifolds”, Topology 27:4 (1988), 513-518. MR 90b:53042 Zbl 0672.53036

[Bieberbach 1911] L. Bieberbach, "Über die Bewegungsgruppen der Euklidischen Räume (erste Abh.)", Math. Ann. 70 (1911), 297-336. JFM 42.0144.02

[Buser and Karcher 1981] P. Buser and H. Karcher, Gromov's almost flat manifolds, Astérisque 81, Société Mathématique de France, Paris, 1981. MR 83m:53070 Zbl 0459.53031 
[Charlap 1965] L. S. Charlap, "Compact flat riemannian manifolds, I", Ann. of Math. (2) 81 (1965), 15-30. MR 30 \#543 Zbl 0132.16506

[Charlap 1986] L. S. Charlap, Bieberbach groups and flat manifolds, Universitext, Springer, New York, 1986. MR 88j:57042 Zbl 0608.53001

[Dekimpe and Igodt 1994] K. Dekimpe and P. Igodt, "The structure and topological meaning of almost-torsion free groups", Comm. Algebra 22:7 (1994), 2547-2558. MR 95f:20057 ZBL 0802. 20041

[Gromov 1978] M. a. Gromov, "Almost flat manifolds", J. Differential Geom. 13:2 (1978), 231-241. MR 80h:53041 Zbl 0432.53020

[Gromov 1981] M. Gromov, "Groups of polynomial growth and expanding maps", Inst. Hautes Études Sci. Publ. Math. 53 (1981), 53-73. MR 83b:53041 Zbl 0474.20018

[Johnson 1990] F. E. A. Johnson, "Flat algebraic manifolds", pp. 73-91 in Geometry of low-dimensional manifolds (Durham, 1989), vol. 1, edited by S. K. Donaldson and C. B. Thomas, London Math. Soc. Lecture Note Ser. 150, Cambridge Univ. Press, Cambridge, 1990. MR 93k:32064 Zbl 0837.57021

[Johnson and Rees 1991] F. E. A. Johnson and E. G. Rees, "Kähler groups and rigidity phenomena", Math. Proc. Cambridge Philos. Soc. 109:1 (1991), 31-44. MR 91i:58040 Zbl 0736.53058

[Lee and Raymond 1985] K. B. Lee and F. Raymond, "Rigidity of almost crystallographic groups", pp. 73-78 in Combinatorial methods in topology and algebraic geometry (Rochester, NY, 1982), edited by J. R. Harper and R. Mandelbaum, Contemp. Math. 44, Amer. Math. Soc., Providence, RI, 1985. MR 87d:57026 Zbl 0575.57026

[Mac Lane 1963] S. Mac Lane, Homology, Grundlehren der math. Wissenschaften 114, Springer, Berlin, 1963. MR 28 \#122 Zbl 0133.26502

[Malcev 1949] A. I. Mal'cev, “On a class of homogeneous spaces”, Izvestiya Akad. Nauk. SSSR. Ser. Mat. 13 (1949), 9-32. In Russian; English translation in Amer. Math. Soc. Translations 39, 1951, 1-33. MR 10,507d

[Milnor 1963] J. Milnor, Morse theory, Annals of Mathematics Studies 51, Princeton University Press, Princeton, N.J., 1963. MR 29 \#634 Zbl 0108.10401

[Milnor 1977] J. Milnor, "On fundamental groups of complete affinely flat manifolds", Advances in Math. 25:2 (1977), 178-187. MR 56 \#13130 Zbl 0364.55001

[Tits 1981] J. Tits, "Groupes à croissance polynomiale (d'après M. Gromov et al.)”, pp. 176-188 in Bourbaki Seminar, 1980/81, Lecture Notes in Math. 901, Springer, Berlin, 1981. MR 83i:53065 Zbl 0507.20015

[Wolf 1968] J. A. Wolf, "Growth of finitely generated solvable groups and curvature of Riemanniann manifolds”, J. Differential Geometry 2 (1968), 421-446. MR 40 \#1939 Zbl 0207.51803

Received June 25 2003. Revised June 52004.

\author{
NiCKOLAS J. MiCHELACAKIS \\ MATHEMATICS AND STATISTICS DEPARTMENT \\ UNIVERSITY OF CYPRUS \\ P.O. Box 20537 \\ Nicosia 1678 \\ CYPRUS \\ njm@ucy.ac.cy
}

